University of Nebraska - Lincoln

DigitalCommons@University of Nebraska - Lincoln

\title{
The Meaning, Selection, and Use of the Peridynamic Horizon and Its Relation to Crack Branching in Brittle Materials
}

\author{
Florin Bobaru \\ University of Nebraska-Lincoln, fbobaru2@unl.edu \\ Wenke Hu \\ University of Nebraska-Lincoln, huwenke_nbu@hotmail.com
}

Follow this and additional works at: https://digitalcommons.unl.edu/mechengfacpub

Bobaru, Florin and Hu, Wenke, "The Meaning, Selection, and Use of the Peridynamic Horizon and Its Relation to Crack Branching in Brittle Materials" (2012). Mechanical \& Materials Engineering Faculty Publications. 81.

https://digitalcommons.unl.edu/mechengfacpub/81

This Article is brought to you for free and open access by the Mechanical \& Materials Engineering, Department of at DigitalCommons@University of Nebraska - Lincoln. It has been accepted for inclusion in Mechanical \& Materials Engineering Faculty Publications by an authorized administrator of DigitalCommons@University of Nebraska Lincoln. 


\title{
The Meaning, Selection, and Use of the Peridynamic Horizon and Its Relation to Crack Branching in Brittle Materials
}

\author{
Florin Bobaru and Wenke Hu
}

\begin{abstract}
Department of Mechanical and Materials Engineering, University of Nebraska-Lincoln, Lincoln, Nebraska, 68588-0526, USA; e-mail fbobaru2@unl.edu
\end{abstract}

\begin{abstract}
This note discusses the peridynamic horizon (the nonlocal region around a material point), its role, and practical use in modeling. The objective is to eliminate some misunderstandings and misconceptions regarding the peridynamic horizon. An example of crack branching in a nominally brittle material (homalite) is addressed and we show that crack branching takes place without wave interaction. We explain under what conditions the crack propagation speed depends on the horizon size and the role of incident stress waves on this speed.
\end{abstract}

Keywords: peridynamics, nonlocal models, continuum mechanics, dynamic fracture, crack branching, brittle fracture

\section{Introduction}

In matters related to material cohesion, physics teaches us that atoms and molecules interact with each other over short distances. These distances, while short, extend beyond the atom's nearest neighbors, especially in solid matter (Israelachvili, 1992). The type of interaction postulated by classical continuum mechanics is, in this view, non-physical, since atoms and molecules are neither infinitesimal in size nor do they interact only via direct contact with their nearest neighbors alone. Nevertheless, classical continuum mechanics has been one of the most successful theories in modeling mechanical behavior of solids and structures for some time now.

The peridynamic formulation of continuum mechanics was introduced in Silling (2000) in order to extend the classical continuum formulation to problems that involve discontinuities, such as cracks. The formulation is based on nonlocal interactions between material points in a continuum. The peridynamic interaction between a material point and its neighbors extends beyond the nearest neighbors, over a region called "the horizon" (see Silling, 2000, and Equation (1) below). A fundamental generalization of the original peridynamic theory beyond pair-wise interaction between material points was published in Silling et al. (2007). A review of peridynamics is given in Silling and Lehoucq (2010). A recent special journal issue (Bobaru, 2011) is dedicated to some aspects of peridynamics and multiscale modeling.

The scope of this note is to clarify the meaning and discuss the selection and use of the peridynamic horizon. In support of this we present a specific example of dynamic crack propagation in a nominally brittle material (homalite) to analyze the dependence of the crack propagation speed on the horizon size. Sudden loading triggering the crack propagation is applied on the faces of the crack, and we show that the crack branches well before interaction with waves reflected from the boundaries takes place. The example shows that peridynamics captures crack branching even when this event is not influenced by stress wave interactions, as has been demonstrated by the experiments of Ravi-Chandar and Knauss (1984). Previ- 
ous results on crack branching with peridynamics were obtained for loadings of the type that led to interactions between stress waves and the propagating crack (see e.g. Ha and Bobaru 2010, 2011a, 2011b, Agwai et al. 2011). With the sudden loading on the crack faces we observe that the crack propagation speed and the time and location of crack branching are independent of the horizon size (a small dependence on the number of nodes covered by the horizon is observed and that is related to the spatial integration accuracy). The propagation speed continues to be largely independent of the horizon size until stress waves (reflected from the boundaries of the sample) return and interact with the propagating crack. From there on, since wave dispersion is determined by the horizon size, the crack propagation speed is influenced by the interaction with the stress waves. The influence of stress waves on the crack propagation speed has been reported in experiments (Ravi-Chandar 2004) and is well captured by peridynamic models (Ha and Bobaru 2010, 2011a, 2011b).

\section{The peridynamic horizon}

The classical continuum mechanics is based on the idea that material points interact only through direct contact with their nearest neighboring points. With the explicit purpose of removing the difficulties classical continuum models faced when dealing with fracture, the peridynamic formulation of continuum mechanics assumes that material points separated by a finite distance interact directly with each other. The origin of such nonlocal interaction is, obviously, the physical interactions between atoms, which extend beyond nearest neighbors. In certain cases these interactions can reach tens of nanometers (Israelachvili, 1992). Moreover, while the interaction atomic potentials drop as the $r^{-6}$ power of the distance $r$ between the atoms, the cumulative effect of molecular forces between nano-structures can drop significantly less rapidly with distance (see Israelachvili, 1992). For example, the potential between two spheres or two cylindrical small-scale structures varies with $D^{-1}$, where $D$ is the distance between the structures, and the potential is proportional to $D^{-2}$ for two parallel surfaces. For newcomers to peridynamics, a major obstacle in understanding it is this notion of a nonlocal interaction between material points. The nonlocal interaction region for a point in the bulk is called the peridynamic horizon, in short, the horizon. The basic formulation for the bond-based peridynamics is:

$$
\rho \ddot{u}(x, t)=\int_{H(x)} f(\tilde{x}-x, u(\tilde{x}, t)-u(x, t)) d \tilde{x}
$$

where $\rho$ is the density, $\ddot{u}(x, t)$ is the acceleration at point $x$ and time $t$, and $f$ is a pairwise force function (per-volume-squared) that depends on the relative position between $\tilde{x}$ and $x$ in some reference configuration and their relative displacement in the current configuration. The nonlocal interaction region for $x$ is $H(x)$. Most commonly, the region is taken to be of spherical/circular shape and the radius of the horizon region is referred to as the horizon size, or simply the horizon (it should be clear from the context whether we refer to the radius of the region, or the region itself). We first note that the peridynamic nonlocal interaction does not necessarily mean or refer to the nonlocal direct physical interaction mentioned above that exists between atoms or small scale structures. The peridynamic horizon may be viewed as an "effective interaction distance" or an "effective length-scale" of a continuum model in a dynamic problem. Notably, in such problems the material length-scale is not necessarily constant, but can depend on the intrinsic dynamics of the problem (e.g. Scheibert et al., 2010). Recent works (Livne et al., 2007, Scheibert et al., 2010) have also identified intrinsic instabilities in dynamic fracture problems and the need for a new characteristic length scale needed to describe these dynamics.

In dynamic fracture problems, "long-range" interaction has been suggested in Ramulu et al. (1983) (see also Streit and Finnie, 1980, Ramulu and Kobayashi, 1985), where a sufficient criterion for crack branching is introduced and tested experimentally. The criterion involves a "characteristic distance $r_{0}$ " that depends on the dynamic state of stress near the crack tip. According to Ramulu et al. (1983), crack 
branching occurs when $r_{0} \leq r_{\mathrm{C}^{\prime}}$ where $r_{\mathrm{c}}$ is a material property thought to be related to the distance between microvoids and the crack tip: when an off-axis microvoid gets within a critical distance $r_{\mathrm{c}}$ from the crack tip, it gets activated by the stress field and directional stability of the propagating crack is lost leading to a deflection of the straight crack path. The loss of directional stability, it is argued in Ramulu et al. (1983), leads, together with the necessary criterion for crack branching $\left(K_{\mathrm{I}} \geq K_{\mathrm{Ib}}\right)$, to branching of a propagating straight crack. The characteristic distance in dynamic fracture is one type of an effectively nonlocal interaction caused by the microstructure of the material (presence of voids). Interestingly, the measured characteristic distance $r_{c}$ is in the range of $0.5-1.5 \mathrm{~mm}$ for the glassy polymers tested (homalite and polycarbonate). This is an effective nonlocal interaction of millimeter size! It is in this sense that the continuum nonlocal interaction, that peridynamics is based on, is to be understood. It is also interesting to point out the, so far unexplored, similarity between the size of the characteristic distance used in Ramulu et al. (1983) and the size of the peridynamic horizon for which peridynamics simulation results (see Ha and Bobaru, 2010 \& 2011a) for crack branching in toughened glass induced by stress waves interaction show cracks reaching propagation speeds that approach values measured experimentally at crack branching. The results in Ha and Bobaru (2010), (2011a), (2011b) as well as in Agwai et al. (2011) were obtained from computational tests in which the dynamic loading is applied from the outer boundaries of the sample, not on the crack faces, and the interaction between the stress waves and the propagating crack influence its growth and its eventual branching. Here we will see that when the dynamic loading is directly applied on the crack surface and no interaction with incident stress waves happens in the time interval monitored, then the crack propagation speed is independent of the horizon size and matches values measured in experiments.

\section{Crack propagation speed and crack branching: The influence of the horizon size}

In peridynamic models, crack branching takes place independent of the horizon size (see Ha and Bobaru (2010)). The propagation speed of a dynamic crack is, however, influenced by the horizon size when the crack propagation event is affected by stress waves, for example waves reflected from the boundaries. Trailing waves behind the main wave (Figure 4 in Ha and Bobaru 2010) are dispersed from it by the nonlocal interaction over the horizon. The magnitude and frequency of these trailing waves depend strongly on the horizon size (see Silling, 2000), and they can induce further breaking of the peridynamic bonds in addition to those broken by the arrival of the main wave front, thus leading to faster propagation speed than measured in experiments (see Ha and Bobaru 2010, 2011a). This behavior is further enhanced in the case of dynamic fracture in anisotropic materials, such as the case of splitting fracture reported in Hu et al. (2011, 2012). Trailing waves caused by dispersion easily break more "matrix-bonds" in unidirectional fiber-reinforced composites and, with a larger horizon size that induces larger amplitude trailing waves than a smaller horizon, the propagation speed is significantly higher than it should be. Once the horizon size is sufficiently small and the strain energy dispersed is reduced, the propagation speed in a splitting crack approaches the measured values (see Hu et al. 2011).

In the example that follows we analyze a case in which there are no interactions between the propagating crack and incident stress waves (other than the "small" waves generated by the breaking of the peridynamic bonds themselves). This is done by applying the dynamic loading on the crack faces and monitoring the event up to the time when the waves reflect from the boundaries and return to influence the propagating crack. This type of loading has been used in experiments (see Ravi-Chandar and Knauss, 1984). We will see that in such situations the propagation speed of a dynamic crack that branches is not affected by the horizon size and is only mildly affected by the spatial integration accuracy (given by the ratio $m=$ horizon/grid-spacing, see e.g. Ha and Bobaru 2010). 


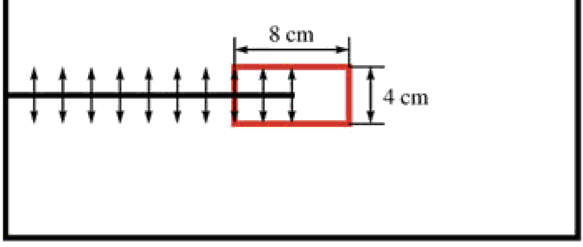

Figure 1. Homalite plate with a pre-crack shock-loaded on crack faces.

The $4 \mathrm{~cm} \times 8 \mathrm{~cm}$ central region is monitored in the results

We consider a two-dimensional pre-cracked homalite sample with dimensions of 20 by $40 \mathrm{~cm}$ as shown in Figure 1 modeled as a brittle peridynamic micro-elastic material. This means that the pairwise force function in Equation (1) is given by an elastic potential

$$
\mathbf{f}(\eta, \xi)=\frac{\partial \omega(\eta, \xi)}{\partial \eta}, \quad \text { with } \omega(\eta, \xi)=\frac{c(\xi) s^{2} \xi}{2},
$$

and where $\xi=\hat{x}-x$ is the relative position in the reference configuration and $\eta=u-\hat{u}$ is the relative displacement for points $\mathbf{x}$ and $\hat{x}$. The bond relative elongation is

$$
\mathrm{s}=\frac{\|\xi+\eta\|-\|\xi\|}{\|\xi\|}
$$

The micro-modulus function $c$ is taken here to be the "conical" function $c(\xi)=c_{1}(1-\|\xi\| / \delta)$ over the horizon. This leads to the following match (see Ha and Bobaru 2010) between the micro-modulus and the elastic modulus:

$$
c_{1}=\frac{24 E}{\pi \delta^{3}(1-v)}
$$

where $E$ is the elastic modulus, $v$ is the Poisson ratio, and $\delta$ is the horizon. Note that in a pair-wise force model, the resulting material has a fixed Poisson ratio of $1 / 3$ in two-dimensions. This limitation is removed in the state-based peridynamics (see Silling et al., 2007). For the purpose of our discussion related to dynamic fracture, this limitation of the bond-based peridynamics is inconsequential. The micro-elastic model is augmented with a damage model that postulates a critical relative elongation for each bond (see Ha and Bobaru, 2011):

$$
s_{0}=\sqrt{\frac{5 \pi G_{0}}{9 E \delta}}
$$

Here $G_{0}$ is the fracture energy for mode I fracture. Material properties for homalite-100 are (Ravi-Chandar 2004): $E=4.55 \mathrm{GPa}, \rho=1230 \mathrm{~kg} / \mathrm{m}^{3}, \mathrm{G}_{0}=38.46 \mathrm{~J} / \mathrm{m}^{2}$.

A loading of $8 \mathrm{MPa}$ is applied suddenly on the crack faces at the beginning of the simulation and is maintained constant during the simulation (see Figure 1). We monitor the crack propagation speed by finding the right-most node in the upper half with a damage index larger than 0.35 . The damage index is a quantity defined for each node as the ratio between the number of broken bonds at the node and number of original bonds at that node. We use a forward difference scheme to estimate the average speed over time intervals of $4 \mu \mathrm{s}$ (data dumps are every $1 \mu \mathrm{s}$ ), and the time-step in all computations is the same, 0.05 $\mu \mathrm{s}$, which is stable for the finest grid used here.

To investigate the effect the nonlocal region size has on the propagation velocity of the dynamic crack, we solve several cases using three different horizon sizes: $4 \mathrm{~mm}, 2 \mathrm{~mm}$, and $1.5 \mathrm{~mm}$, with the corresponding number of nodes of over 80,000,320,000, and 570,000, respectively, for a sample size of 20 by $40 \mathrm{~cm}$. The ratio $m$ between the horizon size and the grid spacing is kept around 4 in most of the simulations. This type of convergence study is called $\delta$-convergence (see Bobaru et al., 2009). In one case, for $\delta=4 \mathrm{~mm}$, we also use $m=8$ to observe the influence of the spatial integration accuracy on the propagation speed. This type of convergence is called $m$-convergence (see Bobaru et al., 2009). 


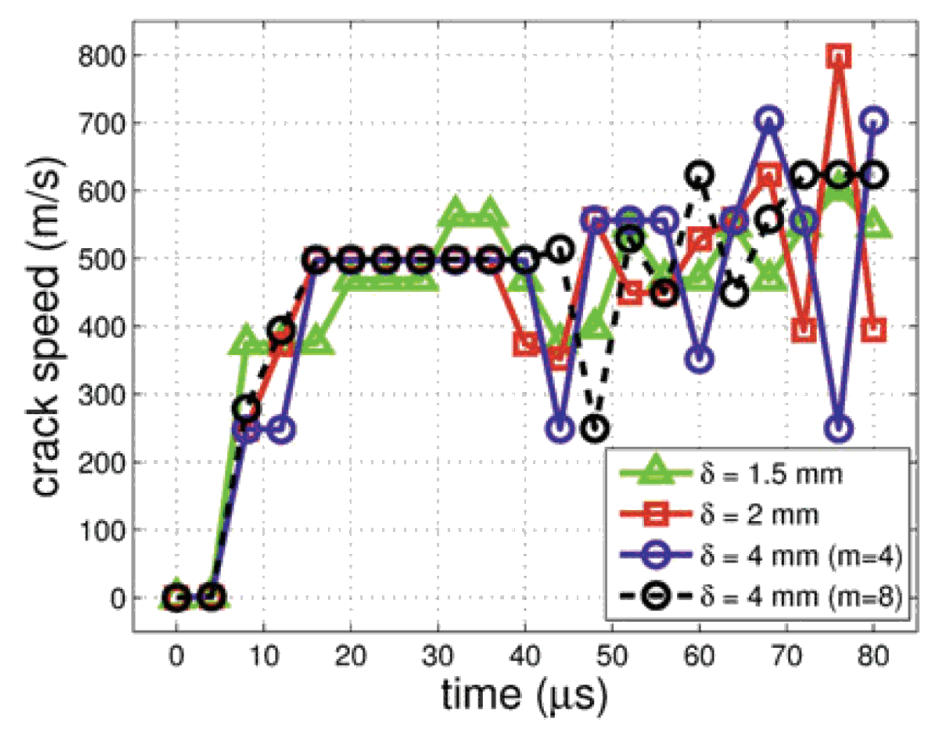

Figure 2. Crack propagation speed in homalite for three different horizon sizes.

The crack propagation speed time-evolution is shown in Figure 2. We observe that the propagation speed is largely unaffected by the size of the horizon. This is in contrast with the results in Ha and Bobaru (2010) and (2011a), and in Hu et al. (2011), where the propagation speed depends on the horizon size, and in which the loading comes from the boundaries and crack propagation is generated by the stress wave interaction. Since now the shock loading is applied on the faces of the pre-notch, such interactions between waves are not taking place, at least not until they return by reflection from the boundaries to meet the propagating crack. The stress waves generated by the suddenly applied load on the pre-crack faces move ahead of the propagating crack.

The rough estimates for the crack branching times (made from visually analyzing the moment damage maps indicate a splitting of the main crack) are around 43-44 $\mu$ s for all horizon sizes, when $m=4$. Branching happens around $47 \mu$ s when $m=8$ (for $\delta=4 \mathrm{~mm}$ ). The crack starts propagating at the same time, speeds up to roughly $0.5 c_{R^{\prime}}$, where $c_{R}$ is the Rayleigh wave speed (1081 m/s in homalite), drops an average of $20 \%$ after branching, and, soon after that, each branch recovers the speed the main crack had before branching. Around $65 \mu$ s the stress waves are returning from the boundaries of the structure and influence the crack propagation speed. More significant oscillations in the crack speed are observed after this time. The average speed before branching is around $500 \mathrm{~m} / \mathrm{s}$, with some better resolution for the smallest horizon size (which also uses the finest discretization of $\Delta \mathrm{x}=0.357 \mathrm{~mm}$, compared to $1 \mathrm{~mm}$ for the $4 \mathrm{~mm}$ horizon and $m=4$ case). This speed is maintained for a considerable time before branching is triggered. When we employ a more accurate spatial numerical integration scheme (for a given horizon size) using $m=8$ for $\delta=4 \mathrm{~mm}$, the only notable difference is the slight delay in the branching time. Other than that, the crack propagation speed is almost identical to that obtained with $\delta=2 \mathrm{~mm}$ and $m=4$, which uses the same spatial discretization grid, and a half-size horizon.

The maximum crack propagation speed before branching in homalite-100 experimentally measured in Ravi-Chandar and Knauss (1984), with the same type of dynamic loading on the crack surfaces used here, was consistently about half of the Rayleigh wave speed, between 400-500 m/s. Most experiments on crack branching (Ramulu and Kobayashi 1985) report a drop in the speed of propagation of about 10\% immediately after branching, followed by a fast recovery to the speed before branching. The crack maintains a propagation speed close to its maximum for a considerable time before branching (Field, 1971). 

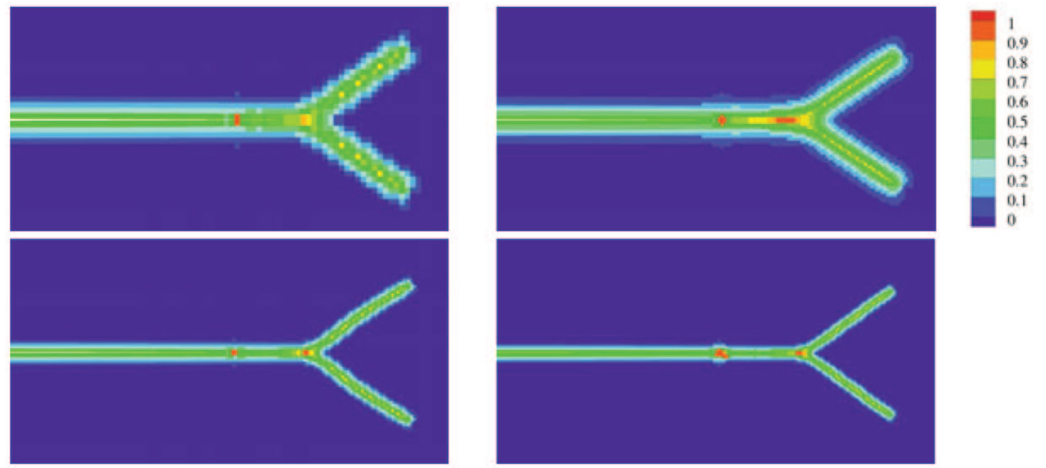

Figure 3. Damage index maps at $80 \mu$ s for the area inside the red line in Figure 1 . Results with three different horizon values: $4 \mathrm{~mm}$ (first row, left with $m=4$, right with $m=8$ ), $2 \mathrm{~mm}$ (second row, left, $m=4), 1.5 \mathrm{~mm}$ (second row, right, $m=4$ ). The same damage index legend is used in all cases.

The angle of branching does not depend on the horizon size, when interaction between the fracture and stress waves is absent, but it does depend on it when the fracture process interacts with stress waves (see Ha and Bobaru 2010, 2011b). Waves can change the propagation path by curving it, and can even arrest a dynamic crack. This is responsible for the way crack branches curve away from, or towards, the main crack, observed in Ha and Bobaru (2010, 2011b) and Agwai et al. (2011).

\section{How to select a "proper" horizon size}

In this section we try to answer the question: "How to choose a proper horizon size in a particular problem?" We suggest the following general guidelines:

a) For cases in which the classical continuum mechanics models have been deemed effective (like in problems in which the material microstructure does not manifests itself, the smaller the horizon the closer the results to the classical ones (since wave dispersion due to the size of the nonlocality is reduced as the horizon decreases). The microelastic peridynamic model or the peridynamic model of heat transfer converge, in the limit of the horizon going to zero, to the corresponding classical models (see Silling and Lehoucq 2008, Bobaru et al. 2009, Bobaru and Duangpanya 2010). Naturally, one would use peridynamic models for problems that are difficult or impossible to solve using the classical models and not for problems for which classical models already provide satisfactory results.

b) For cases in which length-scale effects are apparent (as in the dynamic fracture problems mentioned above or rate-dependent plasticity) or in which the material microstructure and nonlinear, dynamic behavior induce size-effects, a specific horizon size should be used. For example, in dynamic fracture problems the horizon may have to be correlated with the characteristic distance $r_{\mathrm{c}}$ measured in Ramulu et al. (1983), while in problems in which the material microstructure is relevant, the horizon may have to be adjusted to, for example, the average spacing between the inclusions. The original idea proposed in Silling (2000) to adjust the horizon so that the peridynamic results produce the same dispersion curves as those measured for a specific material, was further pursued in Weckner and Silling (2011). A simple example of a derivation of a horizon from an atomistic model was presented for a nonlocal linear spring model in Seleson et al. (2009).

c) For the problem of fracture in a thin plate or a membrane (Silling and Bobaru 2005), in which bending deformation modes or wrinkling may play a significant role in the progression of damage, the horizon size needs to be selected sufficiently small relative to the thickness of the plate/membrane so that bending is not influenced by the size of the nonlocal region at a point. 
d) The peridynamic horizon does not have to be constant over the domain. Examples of how to adaptively scale the horizon around a stress concentration zone are shown with a concurrent model in Bobaru and Ha (2011), while hierarchical coarsening for peridynamics with applications to multiscale modeling are presented in Silling (2011). The hierarchical coarsening approach (Silling 20011) leads to coarsened models with a large horizon, capable of representing the solution with a very fine horizon. In this sense, the peridynamic horizon and nonlocality are viewed as "flexible" ways for building multiscale models.

\section{Conclusions}

The peridynamic horizon is the nonlocal region around a material point in a continuum model that facilitates the replacement of spatial derivatives present in the formulation of a physical phenomenon (mechanical, thermal, etc.) with an integral term. The elimination of spatial gradients is responsible for the ability of the peridynamic theory in modeling phenomena with emerging and evolving discontinuities. The peridynamic horizon can be understood as an effective nonlocal interaction that captures an effective length-scale or nonlocal behavior induced by the material microstructure and/or the type of dynamic loading. A horizon of $1 \mathrm{~mm}$ in a peridynamic model for a dynamic fracture problem does not mean that there exists a physical elastic spring connection between points $1 \mathrm{~mm}$ apart, but rather that the effective nonlocal behavior manifested in the dynamic crack propagation phenomenon is well captured by the peridynamic model with such a horizon size. For problems in which a material length-scale is apparent, the horizon may be correlated to it, and, in general, it has to be taken as small as practically possible for problems where the behavior is well represented by a classical model. This is necessary to reduce the amplitude of the trailing waves dispersed by the nonlocal region. Such dispersive wave influence the evolution of damage, in general leading to cracks that propagate faster than they should since the trailing waves break more peridynamic bonds than an undispersed wave would. We showed that crack propagation and branching can happen in peridynamics without interactions between stress waves and fracture, and that in such cases, the crack propagation speed, crack branching time, and branching location are all independent of the horizon size. A small dependence of the time of branching on the accuracy of spatial numerical spatial integration is observed.

Acknowledgments - Discussions with S. A. Silling, M. L. Parks, R. B. Lehoucq, P. Seleson, and J. T. Foster are gratefully acknowledged. Funds from ARO/ARL (Drs. L. Russell, C. F. Yen, C. Randow), proposal \#58450-EG, and Sandia National Laboratories (Dr. S. A. Silling) supported this work.

\section{References}

Agwai A., Guven, I., Madenci, E. (2011). Predicting crack propagation with peridynamics: A comparative study. Int. J. Fract. 171, 65-78.

Bobaru, F. (2011). Peridynamics and multiscale modeling. Int. J. Multiscale Com. Eng. 9(6), vii-ix.

Bobaru, F., Yang, M., Alves, L. F., Silling, S. A., Askari, E., Xu, J. (2009). Convergence, adaptive refinement, and scaling in 1d peridynamics. Int. J. Numer. Meth. Eng. 77(6), 852-877.

Bobaru, F., Duangpanya, M. (2010). The peridynamic formulation for transient heat conduction. Int. J. Heat Mass Tran. 53(19-20), 4047-4059.

Bobaru, F., Ha, Y. D. (2011). Adaptive refinement and multiscale modeling in 2d peridynamics. Int. J. Multiscale Com. Eng. 9(6), 635-659.

Field, J. E. (1971). Brittle fracture: Its study and application. Contemp Phys 12(1), 1-31.

Ha, Y. D., Bobaru, F. (2010). Studies of dynamic crack propagation and crack branching with peridynamics. Int. J. Fract. 162(1-2), 229-244.

Ha, Y. D., Bobaru, F. (2011a). Characteristics of dynamic brittle fracture captured with peridynamics. Eng. Fract. Mech. 78(6), 1156-1168. 
Ha, Y. D., Bobaru, F. (2011b). Dynamic brittle fracture captured with Peridynamics. Proc. of IMECE 2011, November 11-17, 2011, Denver, Colorado, USA: IMECE2011-65515.

Israelachvili, J. N. (1992). Intermolecular and Surface Forces. Academic Press, second edition.

Livne, A., Ben-David, O., Fineberg, J. (2007). Oscillations in rapid fracture. Phys. Rev. Lett. 98(12), 124301.

Ramulu, M., Kobayashi, A. S., Kang, B. S. J., Barker, D. B. (1983). Further studies on dynamic crack branching. Exp. Mech. 23(4), 431-437.

Ramulu, M., Kobayashi, A. S. (1985). Mechanics of crack curving and branching: A dynamic fracture analysis. Int. J. Fract. 27:187-201.

Ravi-Chandar, K. (1998). Dynamic fracture of nominally brittle materials, Int. J. Fract. 90(1-2), 83-102.

Ravi-Chandar, K. (2004). Dynamic fracture. Elsevier.

Ravi-Chandar, K., Knauss, W. G. (1984). An experimental investigation into dynamic fracture-III. On steady state crack propagation and branching, Int. J. Fract. 26, 141-154.

Scheibert, J., Guerra, C., Célarié, F., Dalmas, D., Bonamy, D. (2010). Brittle-quasibrittle transition in dynamic fracture: An energetic signature, Phys. Rev. Lett. 104(4), 045501.

Seleson, P., Parks, M. L., Gunzburger, M., Lehoucq, R. B. (2009). Peridynamics as an upscaling of molecular dynamics, Multiscale Model. Sim. 8(1), 204-227.

Silling, S. A. (2000). Reformulation of elasticity theory for discontinuities and long-range forces. J. Mech Phys Solids 48(1), 175-209.

Silling, S. A. (2011). A coarsening method for linear peridynamics. Int. J. Multiscale Com. Eng. 9(6), 609-621.

Silling, S. A., Bobaru, F. (2005). Peridynamic modeling of membranes and fibers. Int. J. Nonlinear Mech, 40, 395-409.

Silling, S. A., Epton, M., Weckner, O., Xu, J., Askari, E. (2007). Peridynamic states and constitutive modeling. J. Elast. 88(2), 151-184.

Silling, S. A., Lehoucq, R. (2008). Convergence of peridynamics to classical elast theory. J. Elast. 93, 13-37.

Silling, S. A., Lehoucq, R. B. (2010). Peridynamic theory of solid mechanics. Adv. Appl. Mech. 44, 73-168.

Streit, R., Finnie, L. (1980). An experimental investigation of crack-path directional stability. Exp. Mech. 20(1), 17-23.

Weckner, O., Silling, S. A. (2011). Determination of nonlocal constitutive equations from phonon dispersion relations. Int. J. Multiscale Com. Eng. 9(6), 623-634. 